\title{
Negligencia: modalidad subestimada del maltrato al infante
}

\section{Neglect: underestimated form of child abuse}

\author{
Mónica Rodríguez-González, ${ }^{1}$ Arturo Loredo-Abdalá ${ }^{2}$
}

\begin{abstract}
Resumen
En las últimas dos a tres décadas, el maltrato infantil se ha aceptado mundialmente como una patología grave, que implica alta morbilidad y mortalidad en la víctima, así como su repercusión social y económica en la familia. Entre la tipología del maltrato infantil, la negligencia se reconoce como la forma más frecuente; por su complejidad clínica y social es la de mayor dificultad para establecer su diagnóstico, atención integral y, por ende, registro. Por esto es indispensable insistir y difundir los aspectos clínicos y los sociales básicos: el diagnóstico diferencial con abandono, desamparo, desidia, negligencia social, y otros. Vale la pena hacer hincapié en que las políticas de salud pública deben tener una orientación desde una perspectiva médica, psicológica, epidemiológica, sociológica, criminológica, educativa y económica con fines preventivos. PALABRAS CLAVE: Negligencia; maltrato infantil; prevención

Abstract

In the last 2-3 decades, Child Abuse (CA) has been accepted, worldwide, as a serious pathology, which entails a high morbidity and mortality derived from the physical and emotional outcomes caused in the victim, as well as its social and economic repercussion in the family and society. Within the CA typology, Neglect is recognized as the most frequent form, however, due to its clinical and social complexity, it is also the one that presents the greatest difficulty in establishing a diagnosis, holistic approach and therefore medical record. For this reason, it has been considered essential to emphasize and disseminate the five basic clinical and social aspects, the differential diagnosis with abandonment, homelessness, neglect, social negligence, etc. and insist that public health policies should be oriented from a medical, psychological, epidemiological, sociological, criminological, educational and economic perspective with preventive purposes. KEYWORDS: Neglect; Child Abuse; Prevention
\end{abstract}

\author{
${ }^{1}$ Pediatra. \\ 2 Pediatra internista, especialista en el \\ estudio y atención integral del niño mal- \\ tratado, coordinador de la Coordinación \\ de Estudios Avanzados sobre la Preven- \\ ción del Maltrato Infantil (CEAMI-P). \\ Instituto Nacional de Pediatría, Ciudad \\ de México. \\ Recibido: 1 de junio 2017 \\ Aceptado: 13 de noviembre 2018 \\ Correspondencia \\ Arturo Loredo Abdalá \\ cainm_inp@hotmail.com \\ Este artículo debe citarse como \\ Rodríguez-González M, Loredo-Abdalá \\ A. Negligencia: modalidad subestimada \\ del maltrato al infante. Acta Pediatr \\ Mex. 2019;40(2):85-92.
}

\section{ANTECEDENTES}

En las últimas dos a tres décadas, el maltrato infantil se ha aceptado mundialmente como una patología grave. Es la consecuencia de la magnitud del daño físico-emocional en la víctima y, por la repercusión social y económica en la familia y en su comunidad, en casi todo el mundo. ${ }^{1,2} \mathrm{~A}$ pesar de su morbilidad y mortalidad existe un subregistro de casos porque no se diagnostican correctamente o no se denuncian situaciones que llevan a entender esta modalidad de maltrato infantil en toda su magnitud. ${ }^{3,4}$

\section{Frecuencia}

Por la variabilidad y complejidad del maltrato infantil es muy difícil precisar su frecuencia. En algunos países industrializados quizá sea posible conocer la incidencia y prevalencia de algunas modalidades del maltrato infantil, pero en la mayor parte de las sociedades en vías de desarrollo, el conocimiento de este problema es muy limitado.

En Estados Unidos, como un representante de los países del primer mundo, se reporta un caso 
de maltrato infantil cada diez segundos y se registran, por lo menos, cuatro decesos diarios por algún tipo de maltrato. ${ }^{5} \mathrm{~A}$ pesar de ello, se estima que sólo se reconocen oficialmente $50 \%$ de los casos. ${ }^{6}$ En Inglaterra se reporta una frecuencia global de $1.5 \%$ de casos, en Canadá de $2.15 \%$ y en Australia de $3.34 \%{ }^{3}$

En México las cifras son poco confiables, aunque es muy probable que 1 de cada 10 menores sea víctima de maltrato infantil y solo 1 de cada 100 casos es atendido. ${ }^{7}$ Por el estudio clínico-social de esta patología y sus consecuencias legales, se ha señalado al maltrato infantil como un problema médico-social-legal..$^{8-11}$

Si se entiende y acepta que el maltrato infantil es un problema muy complejo, ello hace que su atención también lo sea. 3, 12,13

Para evitar algunos problemas es necesaria su clasificación y registro como maltrato infantil con sus cuatro grandes modalidades de abuso: físico, sexual, psicológico y negligencia, de acuerdo con la Clasificación Internacional de las Enfermedades (CIE-10). ${ }^{14}$

\section{NEGLIGENCIA}

La negligencia es la forma más frecuente de maltrato infantil, pero por su complejidad clínica y social es la de mayor dificultad para establecer el diagnóstico preciso, atención integral y, por ende, registro. ${ }^{15}$

La Organización de las Naciones Unidas (ONU) considera a la negligencia como un problema multidimensional, que requiere atención multifacética y urgente ${ }^{16}$ porque su repercusión en la salud física y emocional de la víctima es tan grave, como el generado en las otras modalidades de maltrato infantil. ${ }^{17,18}$

La ONU, por medio de la Organización Mundial de la Salud (OMS) y la Organización Panameri- cana de la Salud (OPS) la definen, similarmente, como "...la privación de las necesidades básicas que garantizan al niño un desarrollo bio-psicoafectivo normal cuando existe la posibilidad de brindar alimentación, educación, salud o cuidado". ${ }^{19}$

En Estados Unidos, en la Samoa Americana, Puerto Rico y en las Islas Vírgenes se define como "...la falla de un pariente o un cuidador del menor para cubrir sus necesidades de alimentación, ropa, protección, atención médica o supervisión para cuidar su salud, educación, seguridad y protegerlo de los peligros cotidianos". ${ }^{20}$ La Asociación Afecto, de Colombia, emplea la definición de Polansky que la señala como: "Negligencia es una condición en la que el cuidador responsable de un niño, deliberadamente o por desatención extraordinaria, permite que éste experimente un sufrimiento evitable o fracasa en proveer uno o más ingredientes, generalmente considerados como esenciales, para el adecuado desarrollo físico, intelectual y emocional de las personas". ${ }^{21,22}$ El Colegio Médico del Perú señala como negligencia al: “...maltrato por omisión, en el que se deja de salvaguardar la salud, seguridad y bienestar del niño. Su expresión extrema es el abandono y este puede ser físico, médico, emocional, afectivo o educacional". ${ }^{23}$ En México, la Comisión Nacional de Derechos Humanos considera que negligencia es: "...el fracaso repetido de no proporcionar al niño(a) los estándares mínimos de alimentación, vestido, atención médica, educación, seguridad y satisfacción de sus necesidades físicas y emocionales. Desde una perspectiva legal, se define como "...un ilícito, caracterizado por la omisión en el cuidado, protección y amparo de las personas de la menor edad por lo que se le considera un delito" ${ }^{24,25}$ Cuando una niña, niño o un adolescente sea víctima de un delito, se aplicarán las disposiciones de la Ley General de Víctimas y otras que resulten aplicables. ${ }^{26,27}$ 
La niña, niño o adolescente víctima de negligencia puede sufrir una actitud pasiva o permisiva de los padres o tutores al permitir que otras personas realicen acciones violentas contra ellos. Así mismo, también debe considerarse la sobreprotección y la ausencia del fomento de normas conductuales en los hijos. ${ }^{28}$

También se ha considerado indispensable insistir y difundir los aspectos clínicos y sociales básicos de este problema y señalar el diagnóstico diferencial con abandono, desamparo, desidia, negligencia social, etc. Así, los profesionales que atienden o interactúan con niñas, niños y adolescentes podrán establecer más fácilmente una sospecha diagnóstica, posteriormente el diagnóstico preciso para poner en práctica la atención integral. En el Cuadro 1 se exponen las características básicas de cuatro condiciones que, obligadamente, tienen que ser motivo de un diagnóstico diferencial.

Cuadro 1. Definición de las principales consideraciones diferenciales con negligencia

\begin{tabular}{|l|l|}
\hline \multicolumn{1}{|c|}{ Término } & \multicolumn{1}{c|}{ Definición } \\
\hline Negligencia social & $\begin{array}{l}\text { Falta de cuidado o atención no } \\
\text { intencional a un menor,como } \\
\text { resultado de la pobreza. }\end{array}$ \\
\hline Abandono & $\begin{array}{l}\text { Dejar solo a una niña o niño al } \\
\text { alejarse o privarlo de cuidados, sin } \\
\text { preocuparse por lo que le pueda } \\
\text { suceder. }\end{array}$ \\
\hline Desamparo & $\begin{array}{l}\text { Dejar sin protección a una niña o } \\
\text { niño cuando lo necesita }\end{array}$ \\
\hline Desidia & $\begin{array}{l}\text { Falta de ganas o de interés para } \\
\text { cuidar a una niña o niño }\end{array}$ \\
\hline
\end{tabular}

Academia Mexicana de la Lengua. Diccionario de la Lengua Española.

\section{Cuadro clínico}

Para detectar casos de maltrato infantil es indispensable que el personal de salud (médicos, enfermeras, trabajadores sociales, psicólogos, nutriólogos) maestros y cuidadores o familiares

que están en contacto con personas de este grupo etario, tengan en cuenta que este problema solo se podrá sospechar si se considera que esta modalidad de maltrato infantil es menos clara que otras que, frecuentemente, están inmersas en otras modalidades del maltrato infantil y que puede ocurrir sin importar el nivel socioeconómico, cultural, religioso o educativo. ${ }^{28,29,30}$

La expresión básica de esta modalidad de maltrato infantil es la consecuencia de la acción intencional del cuidador primario, para no cubrir adecuadamente las necesidades biopsicosociales fundamentales de la víctima. ${ }^{19,31}$

Con fines didácticos se proponen las siguientes modalidades de negligencia: ${ }^{30}$

Negligencia en el cuidado del menor.

a. Falla alimentaria

b. Falla en la higiene personal.

c. Falla para usar ropa adecuada.

- Negligencia ambiental

- Negligencia médica

- Negligencia educativa

- Negligencia en la supervisión. Cuadro 2

Debe tenerse en mente que todas estas alteraciones también pueden ser las manifestaciones de abuso físico y psicológico; deterioro del aspecto físico, detrimento de la vestimenta, de la

Cuadro 2. Principales formas de negligencia y su expresión clínica (continúa en la siguiente página)

Negligencia en el cuidado del menor

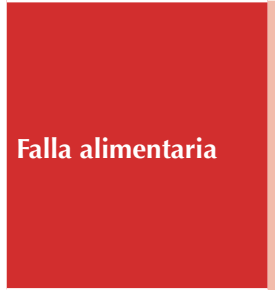

Acciones y manifestación clínica

Para proveer los alimentos necesarios que le permitan al menor satisfacer sus necesidades y requerimientos nutricios o estos son de ínfima calidad nutricional. La expresión clínica puede ser peso y talla bajos para su edad, sobrepeso u obesidad, anémico. 
Cuadro 2. Principales formas de negligencia y su expresión clínica (continuación)

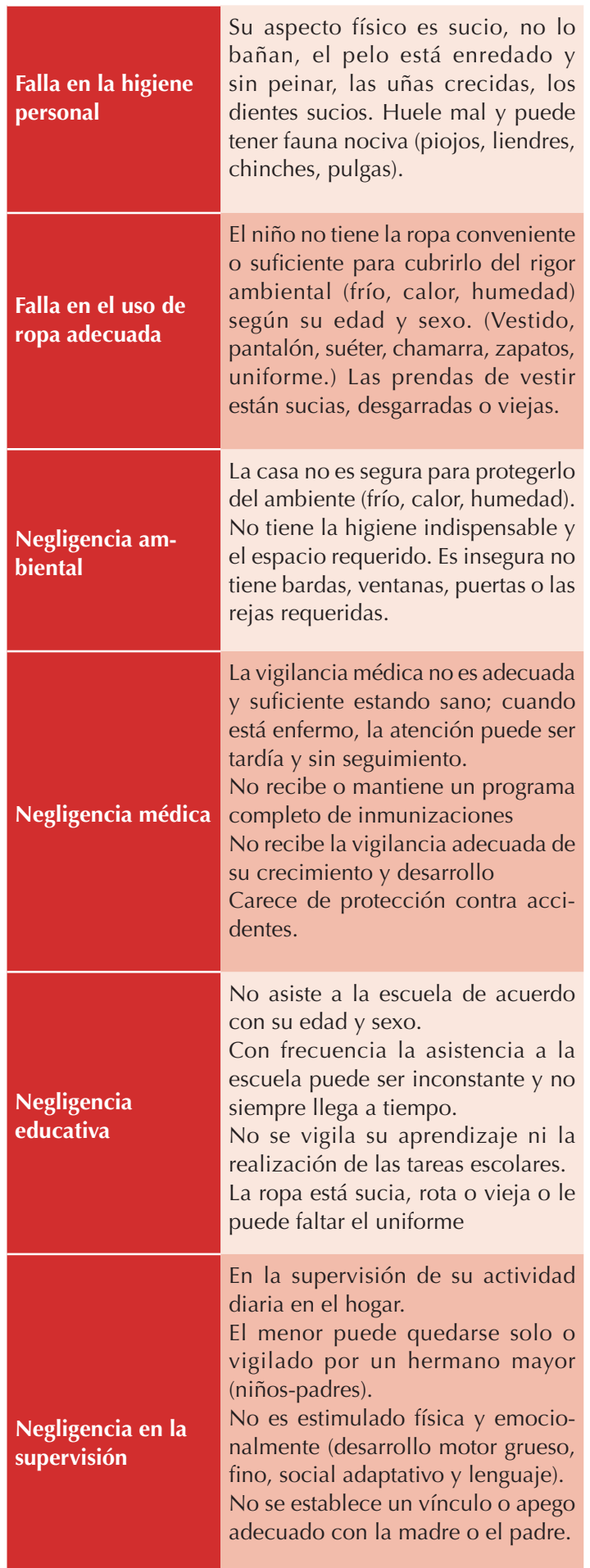

expresión emocional, del rendimiento escolar, de la atención médica y del comportamiento social, entre otras alteraciones que se presentan en el Cuadro 3.

Cuadro 3. Modalidades clínicas, escolares y sociales de negligencia

\begin{tabular}{ll} 
Exposición & Accidentes repetidos. \\
\hline $\begin{array}{l}\text { Antecedentes } \\
\text { patológicos }\end{array}$ & $\begin{array}{l}\text { Retardo en la solicitud de atención } \\
\text { médica. } \\
\text { Mal apego a los tratamientos. }\end{array}$ \\
\hline Grado variable de desnutrición, sobre- \\
peso u obesidad. \\
Detención del crecimiento. \\
Talla baja. \\
Perímetro cefálico disminuido. \\
Retardo en el desarrollo psicomotor.
\end{tabular}




\section{Niveles de gravedad ${ }^{19}$}

La gravedad de la negligencia se cataloga en: leve, moderada y grave.

- Leve cuando la sufre el menor y no le causa problema físico o emocional alguno que altera su vida social.

- Moderada cuando no hay daño físico, pero puede ser la causa directa de rechazo en la escuela, con sus pares del barrio, equipo deportivo, etc.

- Grave cuando la víctima puede sufrir lesiones físicas diversas, como el retardo en el desarrollo intelectual. Por lo tanto, el menor requiere atención y tratamiento específicos.

\section{Diagnóstico diferencial}

Ante algunas de esas manifestaciones, que pueden Ilamar la atención del profesional de la salud, éste está obligado a descartar que no sean consecuencia fundamental de alguna de las "adversidades sociales" como: pobreza, ignorancia, estrategias educativas o disciplinarias inadecuadas, los modelos de crianza (en la familia, la escuela, la iglesia, el barrio) que favorezcan la pérdida de valores o un mal entendido de ellos ${ }^{29-33} \mathrm{o}$, bien, sufrir algún padecimiento pediátrico que pudiera ocasionar un cuadro médico cuya expresión clínica puede ser de desmedro, sobrepeso, obesidad, talla baja, manifestaciones osteo articulares o fracturas repetidas acompañadas de baja autoestima, depresión y angustia, principalmente. ${ }^{34-37}$

La pobreza y la ignorancia señalan claramente la existencia de "negligencia social" cuyos principales responsables de su existencia son los gobiernos o algunas personas de la sociedad civil, siendo una niña, un niño o un adolescente específico, la víctima. Ante un caso así es prioritario iniciar, lo más pronto posible, la atención integral que evite mayor daño físico y emocional en ese menor. ${ }^{37}$

\section{Repercusiones emocionales y económicas a largo plazo}

Una faceta poco considerada en el ambiente médico y paramédico, es la dificultad para establecer cuantitativamente la repercusión social, emocional y económica que genera la atención de una persona que ha sido víctima de negligencia en la familia o en la sociedad. ${ }^{38}$

En la bibliografía internacional está descrito que la repercusión médico-social que sufren o han sufrido las niñas, niños o adolescentes víctimas de negligencia son:

a. Tener 1.5 veces más posibilidades de consumir drogas lícitas (alcohol, tabaco) e ilícitas en la vida adulta.

b. Tener $50 \%$ más de riesgo de embarazarse cuando se es adolescente.

c. Un $25 \%$ pueden delinquir. 39,40

d. El efecto en las funciones cognitivas puede ir desde un grado variable de retraso en el neurodesarrollo o una afectación de grado variable en el rendimiento escolar o académico. $41,42,43$

e. Un efecto tardío en $80 \%$ de los adultos jóvenes pueden ser: la ansiedad, depresión, trastornos de la conducta, agresividad, disminución en el rendimiento académico, ausentismo escolar y problemas con la autoridad. ${ }^{43,44}$

Por último, conocer o precisar el costo económico requerido para la atención integral de víctimas de maltrato infantil varía según lo publicado en países del primer mundo o en los que están en vías de desarrollo. Sin embargo, no se ha publicado un estudio que señale, específicamente, el costo requerido para atender esta modalidad de 
maltrato infantil. Las cifras publicadas permiten establecer una idea de lo económicamente alto que es atender a pacientes víctimas de maltrato infantil. ${ }^{45,46}$

\section{Prevención}

Es evidente que, en general, las víctimas de negligencia requieren de la implementación de una serie de estrategias preventivas de índole familiar, social y gubernamental. La OMS promueve, e insiste, en el desarrollo y evaluación de programas preventivos generales de maltrato infantil, pero no se ha demostrado que alguno de ellos haya alcanzado, a nivel mundial, una eficacia real. Quizá la diversidad de las manifestaciones clínicas, de los contextos sociofamiliares y culturales no permiten igualar las poblaciones, sobre todo en el contexto de las "diversidades sociales". ${ }^{47}$

En México, a partir de 1997, se ha atendido el maltrato infantil de una manera integral en el Instituto Nacional de Pediatría, a través de la Clínica de Atención Integral al Niño Maltratado. Para tener mayor beneficio en la población infantil y juvenil de nuestro país sería muy conveniente su replicación en todos los hospitales pediátricos, considerando que es el único modelo en México que atiende, en su cabalidad, todas sus manifestaciones clínicas. $4,7,8,10,11$

Se insiste que las políticas de salud pública deben tener una orientación desde una perspectiva médica, psicológica, epidemiológica, sociológica, criminológica, educativa y económica con fines preventivos. Solo con la alianza de todas estas disciplinas se podrá aminorar la negligencia y cualquier modalidad de maltrato infantil.

\section{CONCLUSIONES}

Si bien en las últimas tres o cuatro décadas en México se ha mostrado un creciente interés en el reconocimiento del maltrato infantil en cual- quiera de sus cuatro modalidades, es necesario aceptar que poco se ha avanzado en la sospecha, el diagnóstico y en la atención integral de las menores víctimas de negligencia, a pesar de que esta modalidad del maltrato infantil quizá es muy frecuente y su repercusión a mediano o largo plazo, catastrófica.

Es necesario insistir en la necesidad de trabajar en el desarrollo de una intervención dinámica y efectiva de todos los profesionales que atienden o interactúan con niñas, niños o adolescentes, con los padres de familia, maestros y en las diferentes instancias gubernamentales que desarrollan acciones para evitar o aminorar los "determinantes sociales" que afectan la salud física e intelectual de estas víctimas a las que también se les transgreden sus derechos que impidan alcanzar su máximo potencial físico e intelectual a corto, mediano o largo plazo.

\section{REFERENCIAS}

1. Gilbert R, et al. Recognizing and responding to child maltreatment. Lancet. 2009; 373:167-80. https://doi. org/10.1016/S0140-6736(08)61707-9

2. Dubowitz $\mathrm{H}$, et al. Physical abuse and neglect of children. Lancet. 2007; 369:1891-9. https://doi.org/10.1016/S01406736(07)60856-3

3. Gilbert R, et al. Burden and consequences of child maltreatment in high-income countries. Lancet. 2009; 373: 68-80. https://doi.org/10.1016/S0140-6736(08)61706-7

4. Loredo-Abdalá A. Maltrato en niños y adolescentes. Ciudad de México: Editores de Textos Mexicanos, 2004.

5. US Department of Health and Human Services Administration for Children and Families Administration on Children, Youth and Families Children's Bureau (2011). Child Abuse and Neglect Fatalities 2011: Statistics and Interventions.

6. Rovi S, Chen P, Johnson MS. The economic burden of hospitalizations associated with child abuse and neglect. 2004;94(4):586-90.

7. Loredo-Abdalá A, Monroy-Llaguno DA, Casas-Muñoz A. El maltrato infantil: Conceptos básicos de una patología médico social legal. Rev Fac Med UNAM 2013;56(6):5-10.

8. Loredo Abdalá A, Casas Muñoz A, Monroy Llaguno DA. Maltrato infantil. Expresión clínica predominante de pacientes atendidos en el Instituto Nacional de Pediatría (México). PRECOP-SCP; 13: 40-45 
Rodríguez-González M, et al. Negligencia: modalidad subestimada del maltrato al infante

9. Loredo Abdalá A, Trejo Hernández J, García Piña C, PortilloGonzález A, et al. Maltrato infantil: una acción interdisciplinaria en México. Comisión Nacional para el Estudio y la Atención Integral al niño maltratado. Primera parte. Salud Mental. 2010; 33:281-90.

10. Loredo Abdalá A. Maltrato al menor: consideraciones clínicas sobre maltrato físico, agresión sexual y deprivación emocional. Gac Med Méx. 1999; 135:611-20.

11. Loredo-Abdalá A, Casas-Muñoz A, Monroy-Llaguno DA. Maltrato infantil: características clínicas usuales. Rev Fac Med UNAM. 2014;57(1):15-23.

12. Corchado CHS, Loredo-Abdalá A, Perea-Martínez A, Villa RA. El niño maltratado: obstáculos médico-legales en el diagnóstico y asistencia integral. Bol Med Hosp Infant Mex. 2004;61(5):412-22.

13. Loredo-Abdalá A, et al. Children injured: abuse or accident? Diagnosis through indicators. Bol Med Hosp Infant Mex. 2003; 60(4): 368-79.

14. Paul AR, Adams MA. Non-accidental trauma in pediatric patients: a review of epidemiology, pathophysiology, diagnosis and treatment. Trans Pediatr 2014;3:195-207. doi: 10.3978/j.issn.2224-4336.2014.06.01

15. Loredo-Abdalá A, et al. Child abuse: risk factors associated with the macro and microenvironment in a suburban zone within Mexico City. Bol Med Hosp Infant Mex 2003; 60(3): 252-262.

16. Clasificación Internacional de Enfermedades (CIE-10). Revisión. Organización Panamericana de la Salud-Organización Mundial.

17. Allin H, Wathen CN, Macmillan H. Treatment of child neglect: a systematic review. Canad J Psychiatr. 2005; 50:497-504.

18. Sedlak AJ, Mettenburg J, Basena M, et al. Fourth National Incidence Study of child abuse and neglect (NIS-4). Washington, DC: US Department of Health and Human Services; 2010.

19. Maltrato Infantil: una acción interdisciplinaria e interinstitucional en México. Consenso de la Comisión para el Estudio y la Atención Integral al Niño Maltratado. Segunda parte. Salud mental. 2011;34:67-73.

20. Runyan DK, et al. Children who prosper in unfavorable environments the relationship to social capital. Pediatrics 1998;101:12-18.

21. Casas Muñoz A. Negligencia: la forma más frecuente e inadvertida del maltrato infantil. En: Loredo Abdalá A. Maltrato Infantil: Gravedad y prevención. México: Editores de Textos Mexicanos, 2016.

22. Child Welfare Information Gateway. Definitions of child abuse and neglect. Washington, DC: U.S. Department of Health and Human Services, Children's Bureau. 2014.

23. Polansky NA, et al. The psychological ecology of the neglectful mother. Child Abuse and Neglect. 1985; 9:265-275.

24. Gaudin JM, et al. Family functioning in neglectful families. Child Abuse and Neglect.1996;20:363-377.
25. Del Águila-Escobedo A. Violencia y estrés infantil: ¿está en juego nuestro futuro? Acta Médica Peruana. 2015; 32:71-83.

26. Código Penal Federal (2009), Artículos 335 y 339.

27. Ley General de Víctimas. Ley publicada en la Primera Sección del Diario Oficial de la Federación. Febrero, 2013.

28. Modelo Integral de Atención a Víctimas. Diario Oficial de la Federación. Junio 2015.

29. Olivien G. Évaluation du rattrapage de la croissance chez les garçons d'âge préscolaire ayant souffert pendant une longue période de négligence et de maltraitance psychologique. Child Abuse \& Neglect. 2003; 103-108.

30. INEGI. Mujeres y hombres en México. 2014-2015.

31. Perea Martínez A, Loredo Abdalá A, López Negrete GE y Jordán GN. ¿Negligencia o pobreza? El sobrediagnóstico de maltrato al menor. Acta Pediatr Mex. 2007; 28:193-97.

32. UNICEF-CONEVAL, Pobreza y Derechos Sociales de Niños, Niñas y Adolescentes en México, 2010-2012.

33. Gutiérrez JP, et al. Encuesta Nacional de Salud y Nutrición 2012. Resultados Nacionales. Cuernavaca, México: Instituto Nacional de Salud Pública (MX), 2012.

34. X Consejo Nacional de Evaluación de la Política de Desarrollo Social. Informe de pobreza en México, 2012. México, DF: CONEVAL, 2013.

35. Veilleux LN, et al. The functional muscle-bone unit in patients with osteogenesis imperfecta type I. Bone. 2015; 79:52-57. https://doi.org/10.1016/j.bone.2015.05.019

36. Kolker $\mathrm{S}$, et al. Diagnosis and management of glutaric aciduria type I - revised recommendations. J. Inherit. Metab. Dis. 2011;34:677-94. doi: 10.1007/s10545-011-9289-5

37. Velásquez Jones L, Medeiros Domingo M. Raquitismos hipofosfatémicos hereditarios. Bol Med Hosp Infant Mex. 2013; 6:421-31.

38. Ethier LS, et al. Effet de la durée, de l'âge d'exposition à la négligence parentale et de la comorbidité sur le développement socioémotionnel à l'adolescence Neuropsychiatrie de l'enfance et de l'adolescence. 2009;57:136-45. https:// doi.org/10.1016/j.neurenf.2008.12.004

39. Dahlberg LL, Krug EG. World Report on Violence and Health. Geneva, Switzerland: World Health Organization; 2002:1-56.

40. Embarazo adolescente y madres jóvenes en México: Una visión desde el Promajoven. 1a ed. Secretaría de Educación Pública. México, 2012; 1-185

41. Belitzky R. Resultados Perinatales en Madres Jóvenes: Estudio Comparativo en Maternidades Latinoamericanas. En: La salud del Adolescente y el joven en las Américas. Washington, DC: OPS/OMS. 1985.

42. Villalobos-Hernández $\mathrm{A}$, et al. Embarazo adolescente y rezago educativo: análisis de una encuesta nacional en México. Salud Pública de Méx. 2015; 57:135-43.

43. Trickett PK, McBride-Chang C. The Developmental Impact of Different Forms of Child Abuse and Neglet. Dev Rev. 1995; 15:311-37. 
44. Chapple CL, et al Child neglect, social context, and educational outcomes: examining the moderating effects of school and neighborhood context. Violence and Victims. 2010; 25:470-85. doi: 10.1891/0886-6708.25.4.470

45. Eckenrode J, et al. School performance and disciplinary problems among abused and neglected children. Developmental Psychology. 1993; 29:53-62.
46. Fantuzzo J W, et al. Types and timing of child maltreatment and early school success: A population-based investigation. Children and Youth Services Review. 2011; 33:1404-11. https://doi.org/10.1016/j.childyouth.2011.04.010

47. Messman-Morre $T$, et al. Emotion dysregulation and risky sexual behavior in revictimization. Child Abuse \& Neglect. 2010;34:967-76. https://doi.org/10.1016/j. chiabu.2010.06.004 